\title{
Causas de variação nos preços de bovinos nelore elite no Brasil
}

\author{
Causes of variation in the prices of nelore elite cattle in Brazil
}

\begin{abstract}
João Cláudio do Carmo Paneto ${ }^{\mathrm{I}^{*}}$ Eustáquio Resende Bittar ${ }^{\mathrm{I}}$ Eugênio Faria Barbosa ${ }^{\mathrm{II}}$ Carina Diniz Rocha" José Eduardo do Val ${ }^{\mathrm{III}}$ Antônio Sergio Ferraudo ${ }^{\mathrm{IV}}$ Raysildo Barbosa Lôbo $^{\mathrm{III}, \mathrm{V}}$
\end{abstract}

\section{RESUMO}

Este trabalho teve por objetivo estudar as causas de variação nos preços de bovinos da raça nelore pertencentes a rebanhos de seleção, os quais foram comercializados em leilões, para verificar as influências das avaliações genéticas $e$ dos julgamentos de exterior sobre esses preços. Para tanto, foram computados os preços de venda de 426 bovinos da referida raça em 12 leilões ocorridos em diversas localidades brasileiras (regiões Centro-Oeste, Norte e Sudeste), entre os anos de 2002 e 2005. O valor médio foi de $R \$ 3.325,49$, sendo o mínimo de $R \$ 1.400,00$ e o máximo de $R \$ 10.500,00$. Esses dados foram digitados juntamente com outras informações que eram apresentadas nos catálogos dos leilões. As informações registradas incluíram o sexo de cada animal, o nome do leilão e as DEPs informadas nos catálogos. Além da avaliação da influência das informações dos catálogos, também foi avaliada a influência das informações dos reprodutores, pais dos animais vendidos nos leilões, envolvendo suas DEPs publicadas em um sumário de reprodutores da raça e as pontuações de suas progênies em julgamentos. Os métodos estatísticos aplicados foram análises de variâncias $e$ análises de agrupamento (método K-médias). Como resultado, foi observado que animais com superioridade genética em características relacionadas a desempenho ponderal, considerando-se os efeitos diretos e maternos, foram valorizados ao serem comercializados nos leilões. Em contra-partida, a pontuação dos reprodutores nos julgamentos não teve influência significativa sobre os preços médios de venda de suas progênies nos leilões.
Palavras-chave: bovinocultura de corte; preços; leilões; DEP; julgamento.

\begin{abstract}
This study aimed to understand the causes of variation in the marketing prices of elite flock nelore cattle commercialized by auction, especially to verify the influences of EPDs and visual assessment. The selling prices of 426 animals from the nelore breed commercialized during 12 auctions held in various Brazilian locations, between the years 2002 and 2005, were considered in the analysis. The average price was $R \$ 3.325,49$, with a minimum of $R \$ 1.400,00$ and a maximum of $R \$ 10.500,00$. The data used in a first analysis included the sex of each animal, the name of the auction and the EPDs of each animal informed in a catalog provided to the buyers during the auctions. A second analysis considered the EPDs of the sires of the commercialized animals, and the accumulated punctuation of these sires through their progeny judged through visual assessment during traditional animal shows in Brazil. Variance analysis and $k$-means clustering were applied to run the statistical analysis of the data. It was observed that animals with better EPDs, direct and maternal effects, on growth traits were sold with higher prices during the auctions. The punctuation by visual assessment did not significantly affect the prices.
\end{abstract}

Key words: beef cattle, prices, auctions, EPD, visual assessment.

\footnotetext{
'Universidade de Uberaba, Faculdades Associadas de Uberaba, Associação Brasileira dos Criadores de Zebu (UNIUBE/FAZU/ABCZ), Curso de Med. Veterinária. Av. Nenê Sabino, 1801, 38055-500, Uberaba, MG, Brasil. E-mail: paneto@yahoo.com. *Autor para correspondência.

"UNIUBE, Uberaba, MG, Brasil.

"I'Departamento de Genética, Faculdade de Medicina de Ribeirão Preto, Universidade de São Paulo (FMRP/USP), Ribeirão Preto, SP, Brasil.

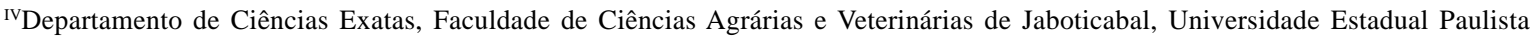
(FCAV/UNESP), Jaboticabal, SP, Brasil.

vesquisador Associado da Associação Nacional de Criadores e Pesquisadores (ANCP), Ribeirão Preto, SP, Brasil.
} 


\section{INTRODUÇÃO}

Os programas de melhoramento genético no Brasil têm obtido avanços significativos, trazendo aumento na produtividade e diminuição nos custos da produção comercial de bovinos de corte. A longo prazo, esses avanços podem propiciar a diminuição dos preços e o aumento da disponibilidade de alimentos, o que faz desses programas elementos de grande importância social. Entretanto, sabe-se que tais avanços não atingem todos os rebanhos simultaneamente e aqueles que se destacam, conseguindo diminuir seus custos de produção e aumentar sua produtividade antes dos demais, certamente serão beneficiados com maiores lucros nesse momento. É essa perspectiva que faz com que os produtores estejam dispostos a pagar mais por animais de valores genéticos superiores, em comparação com os preços de comercialização comuns (BICHARD, 1996).

Uma correta previsão dos resultados esperados é uma maneira de qualquer empresa otimizar suas decisões e errar menos, melhorando conseqüentemente o seu desempenho e garantindo sua competitividade no mercado. A informação das estimativas de valores genéticos de cada animal, na forma de DEP (Diferença Esperada na Progênie), em cada característica de interesse, pode ser usada como uma ferramenta de grande importância na predição do desempenho futuro dos descendentes dos animais utilizados hoje como reprodutores. ADEP, que expressa o valor genético dos animais avaliados, minimiza os possíveis erros que possam ser cometidos no processo de escolha dos reprodutores (LÔBO, 1999).

Tomando consciência da importância dessa ferramenta, os criadores de bovinos já estão utilizando a DEP como informação essencial na hora da compra e venda dos animais. Dessa forma, espera-se que estejam dispostos a pagar mais por animais com melhores valores de DEP nas características de interesse. PEREIRA (1998) comenta que é crescente o nível de exigência por dados que possam predizer o desempenho de um reprodutor. Destaca-se, assim, uma conseqüência comercial das avaliações genéticas - o aumento dos preços dos animais com estimativas desejáveis de valores genéticos para características de interesse.

Apesar dessa emergente "consciência” no mercado de compra e venda de animais de seleção, outros fatores, não ligados às avaliações genéticas, ainda podem afetar muito os preços, principalmente quando se trata da comercialização realizada por meio de leilões. O próprio fato de o animal estar sendo comercializado em um leilão que historicamente vende animais a preços altos pode aumentar o seu preço. Por esse motivo, as diferentes causas de variação nos preços dos animais de seleção precisam ser mais bem compreendidas.

Ao avaliar o ponto de vista do selecionador, que pretende vender seus produtos, percebe-se que há vários fatores influenciando a direção e o tamanho das mudanças genéticas nos rebanhos, dentre os quais, a efetividade dos sinais de mercado dentre os membros da cadeia produtiva (AMER, 2003). A valorização de animais geneticamente superiores, que acontece pelo conhecimento do potencial de produção esperado de seus descendentes, é também um sinal de mercado, se tornando um importante estímulo para que os criadores participem de programas de melhoramento e façam seleção utilizando os resultados das avaliações genéticas. Portanto, um fato reforça o efeito do outro. Os animais geneticamente selecionados são valorizados na hora da comercialização, e essa valorização estimula os criadores a fazerem seleção de forma efetiva, utilizando a tecnologia disponível e gerando animais ainda superiores.

A avaliação de características do exterior dos animais também é uma ferramenta importante e usual na seleção de bovinos. Geralmente, durante eventos de exposição, os animais são avaliados por juízes treinados e credenciados pela Associação Brasileira dos Criadores de Zebu (ABCZ). No entanto, a correlação entre os resultados dos julgamentos e o perfil genético dos animais em características de interesse econômico ainda não está bem definida.

Alguns autores pesquisaram as correlações entre características de exterior e características economicamente relevantes. GUTIÉRREZ et al. (2002) estudaram as relações genéticas entre data do parto, intervalo entre partos, idade ao primeiro parto e características de tipo em gado de corte na Espanha. Esses autores comentam que há muito tempo os criadores acreditam que características de tipo podem ser bons indicadores do desempenho e por isso a observação do exterior é uma atividade comum na maioria dos programas de melhoramento. KOURY FILHO et al. (2003) estudaram os parâmetros genéticos de escores de umbigo e suas correlações com características de desenvolvimento ponderal na raça nelore, entendendo que essa característica de exterior está ligada a traumas e outras patologias reprodutivas em machos criados a pasto. HORIMOTO (2005) fez estimativas de parâmetros genéticos para escores de estrutura corporal (frame) em bovinos, comparando duas metodologias para obtenção dos escores e suas correlações com características consideradas em avaliações genéticas da raça nelore. Além disso, 
GUTIÉRREZ et al. (2002) comentam que a classificação por tipo é difícil de ser conduzida porque usualmente as condições de manejo são extensivas. Ademais, os escores (pontos) usados para classificação podem considerar animais que apresentam condições extremamente ruins em algum quesito ou animais que estejam simplesmente abaixo da média, levando à perda da relação biológica entre as características. Isso pode acontecer quando os critérios de pontuação não são capazes de detectar toda variabilidade existente em cada característica observada.

Historicamente, os conceitos relacionados à estrutura corporal de bovinos de corte seguiram tendências ora ditadas por modismos da raça, ora por exigência dos sistemas produtivos. Hoje, a utilização de animais adequados ao sistema produtivo e às preferências de mercado, sejam elas quais forem, é um aspecto determinante para viabilizar a eficiência econômica da empresa pecuária e condição primordial para a permanência na atividade (HORIMOTO, 2005).

O presente trabalho teve o objetivo de avaliar as influências dos julgamentos de exterior e das estimativas de DEP para características de importância econômica sobre os preços de venda de bovinos da raça nelore, provenientes de rebanhos de seleção em diversas regiões do Brasil.

\section{MATERIAL E MÉTODOS}

Análise dos dados dos catálogos e preços

Em uma primeira etapa, a valorização dos animais de seleção foi estimada a partir dos preços de venda dos animais em leilões. Para tanto, foram computados os preços de 426 animais vendidos em 12 leilões, ocorridos em diversas localidades brasileiras (regiões Norte, Centro-Oeste e Sudeste), entre os anos de 2002 e 2005. Esses dados foram digitados em planilha do MS-Excel ${ }^{\circledR}$ juntamente com algumas estimativas de DEP (Diferenças esperadas nas progênies), e outras informações relevantes que estavam presentes nos catálogos fornecidos aos compradores no início dos leilões, que foram: sexo dos animais em cada lote; nome do leilão; cidade; estado; e ano em que ocorreu o leilão.

As características para as quais as estimativas de DEP de todos os 426 animais eram conhecidas incluíram: efeito maternal para peso aos 120 dias de idade (MP120); peso aos 120 dias de idade (DP120); peso aos 450 dias de idade (DP450); e perímetro escrotal aos 450 dias de idade (PE450).

A partir de uma análise de variâncias, foram avaliadas todas as prováveis fontes de variação, dentre elas, os efeitos das DEPs, testadas primeiramente de forma individual e depois simultânea, até se chegar ao modelo estatístico que melhor explicasse as variações dos preços. O modelo estatístico geral aplicado nessa análise foi:

$\mathrm{Y}_{\mathrm{ijkl}}=\mathrm{SL}_{\mathrm{ij}}=\mathrm{DEP}_{\mathrm{k}}=\mathrm{E}_{\mathrm{ijk}}$

em que:

$\mathrm{Y}_{\mathrm{ijkl}}=$ preço de venda de cada animal;

$\mathrm{SL}_{\mathrm{ij}}=$ efeito fixo da i-ésima categoria de sexo e leilão;

$\mathrm{DEP}_{\mathrm{k}}=$ efeito aleatório associado ao valor de cada uma DEPs;

$\mathrm{E}_{\mathrm{ijkl}}=$ erro aleatório associado a cada observação;

Utilizando-se as variáveis categóricas do modelo da análise de variâncias, um modelo de regressão múltipla foi aplicado para estimar os valores preditos para cada sexo em cada leilão. As diferenças entre os preços preditos e os observados foram chamadas de resíduos. Essas diferenças foram analisadas por técnica de agrupamento k-médias. As análises de agrupamento têm o objetivo de classificar uma amostra de indivíduos em um pequeno número de grupos mutuamente excludentes, com base nas similaridades entre os indivíduos (HAIR et al., 2005). VAL et al. (2008) apresentam uma descrição mais detalhada da técnica de agrupamento k-médias. Todos os valores foram padronizados antes da realização das análises de agrupamento e optou-se pela formação de três grupos para realização do comportamento dos resíduos, quando observados em relação aos valores genéticos em cada característica.

Análise das pontuações dos julgamentos

Além da análise que avaliou a valorização apenas por meio dos dados constantes dos catálogos, na outra abordagem foi analisada a influência dos resultados dos reprodutores em julgamentos sobre os preços médios de venda de suas progênies nos leilões. Para tanto, foram utilizados os resíduos obtidos na análise de regressão, agrupados por reprodutor e pontuações médias dos reprodutores, obtidas por meio de suas progênies avaliadas em julgamentos ocorridos na cidade de Uberaba, Minas Gerais (MG), nos anos de 2004 e 2005. Foram calculadas as médias de pontos por progênie exposta de 92 touros. Essas médias foram atribuídas aos reprodutores que tiveram no mínimo duas progênies comercializadas em leilões, analisandose a correlação entre os preços das progênies nos leilões e os pontos dos reprodutores em julgamentos.

Todas as análises estáticas foram realizadas com o uso do software Statistica 7.1 (STATSOFT, 2003).

\section{RESULTADOS E DISCUSSÃO}

Análise dos dados dos catálogos e preços

Os animais considerados na análise foram vendidos a um preço médio de R \$3.325,49, com mínimo 
de R \$1.400,00 e máximo de R \$10.500,00. Nas análises de variâncias, o modelo que incluía as categorias de sexo e leilão e as estimativas de diferenças esperadas nas progênies MP120, DP120, e DP450 apresentou um coeficiente de ajuste $R^{2}=0,34$. Assim, pouco mais de um terço das causas de variação nos preços puderam ser explicadas pelo modelo estatístico, enquanto os outros dois terços foram intangíveis. Considerandose que a variável em questão (preço) depende, no momento de cada leilão, da disposição dos compradores presentes em pagar mais ou menos pelos animais, podese dizer que o entendimento de um terço de suas causas de variação já é uma informação importante. Todas as causas de variação incluídas no modelo foram significativas $(\mathrm{P}>0,05)$.

A maioria dos leilões estudados comercializou somente animais do sexo masculino. No entanto, nos leilões em que havia machos e fêmeas, os dois sexos apresentaram médias de preços significativamente diferentes entre si, havendo leilões em que os machos foram mais caros e leilões em que as fêmeas foram mais caras. Foi observado que as diferentes categorias de sexos nos diferentes leilões apresentaram diferenças significativas entre si.

Animais com estimativas superiores para os efeitos genéticos diretos e maternos para peso aos 120 e para os efeitos diretos para peso aos 450 dias de idade obtiveram melhores preços de comercialização nos leilões estudados.

No modelo de regressão, ambas as variáveis categóricas - sexo e leilão - foram significativas $(\mathrm{P}<0,01)$ na predição dos preços, e o modelo apresentou um coeficiente de ajuste igual a 0,28 . Assim, o modelo de regressão permitiu o ajuste dos dados de acordo com essas duas variáveis que explicavam aproximadamente $28 \%$ da variação nos preços. Os resíduos obtidos foram considerados independentes desses fatores e, por isso, adequados para a análise de agrupamento.

$\mathrm{Na}$ análise de agrupamento, foram considerados os resíduos e as informações de estimativas de diferenças esperadas nas progênies
(MP120, DP120 e DP450). Todas as variáveis foram significativas, como se pode verificar pelos dados detalhados na tabela 1.

O grupo 1 foi formado por 160 animais, apresentando média superior para os valores dos resíduos, ou seja, com preços mais elevados em relação às predições do modelo de regressão, que já descontavam os efeitos de leilão e sexo. $O$ grupo 2 foi formado por 107 animais, apresentando médias intermediárias para os valores dos resíduos. O grupo 3 foi formado por 159 animais, apresentando médias inferiores para os valores dos resíduos. Essas informações estão ilustradas por meio da figura 1.

Analisando-se as médias dos grupos nas variáveis estudadas, foi possível perceber que os melhores preços - atingidos pelo grupo 1 - estiveram associados ao desempenho superior dos animais nas estimativas de efeito genético direto para os pesos aos 120 e aos 450 dias de idade. Observou-se ainda que 0 grupo 2 - de preços intermediários - foi composto por animais que se destacaram nas estimativas de efeitos genéticos maternais para peso aos 120 dias. Já os piores preços foram obtidos pelo grupo 3 , que apresentou médias inferiores em todas as estimativas de valores genéticos consideradas.

Em um estudo sobre os valores econômicos relativos das características a serem incluídas nos objetivos de seleção em várias espécies, inclusive bovinos com aptidão para corte, WOLFOVÁ \& NITTER (2004) concluíram que os efeitos maternais não deveriam ser negligenciados em rebanhos de raças puras, indicando que tais efeitos deveriam receber entre 0,55 e 0,60 da ponderação dos efeitos diretos. No presente estudo, pôde-se perceber que, mesmo sem o conhecimento dos verdadeiros valores econômicos das características, muitos compradores de gado de elite valorizaram também os efeitos maternos, acertando ao menos parcialmente em seus critérios de escolha.

FERNÁNDEZ-PEREA \& ALENDA JIMÉNEZ (2004) estimaram os valores econômicos relativos das características a serem incluídas no

Tabela 1 - Análise de variâncias indicando os fatores relevantes na formação dos grupos da análise de agrupamento, incluindo fontes de variação (FV), somas de quadrados (SQ), graus de liberdade (gl), valores calculados (F) e níveis de significância (P) dos fatores que influíram nos preços dos animais leiloados.

\begin{tabular}{lcccccc}
\hline Fator / FV & SQ entre grupos & gl & SQ dentro dos grupos & gl & F & P \\
\hline Resíduo & 52,5 & 2 & 372,5 & 423 & 29,8 & 0,00 \\
MP120 & 218,4 & 2 & 206,6 & 423 & 223,4 & 0,00 \\
DP120 & 223,8 & 2 & 201,2 & 423 & 235,3 & 0,00 \\
DP450 & 219,8 & 2 & 205,2 & 423 & 226,5 & 0,00 \\
\hline
\end{tabular}

Resíduo: Diferença entre o preço predito pelo modelo prévio de regressão e o preço real; MP120: efeito genético materno para peso aos 120 dias de idade; DP120: efeito genético direto para peso aos 120 dias de idade; DP450: efeito genético direto para peso aos 450 dias de idade. 


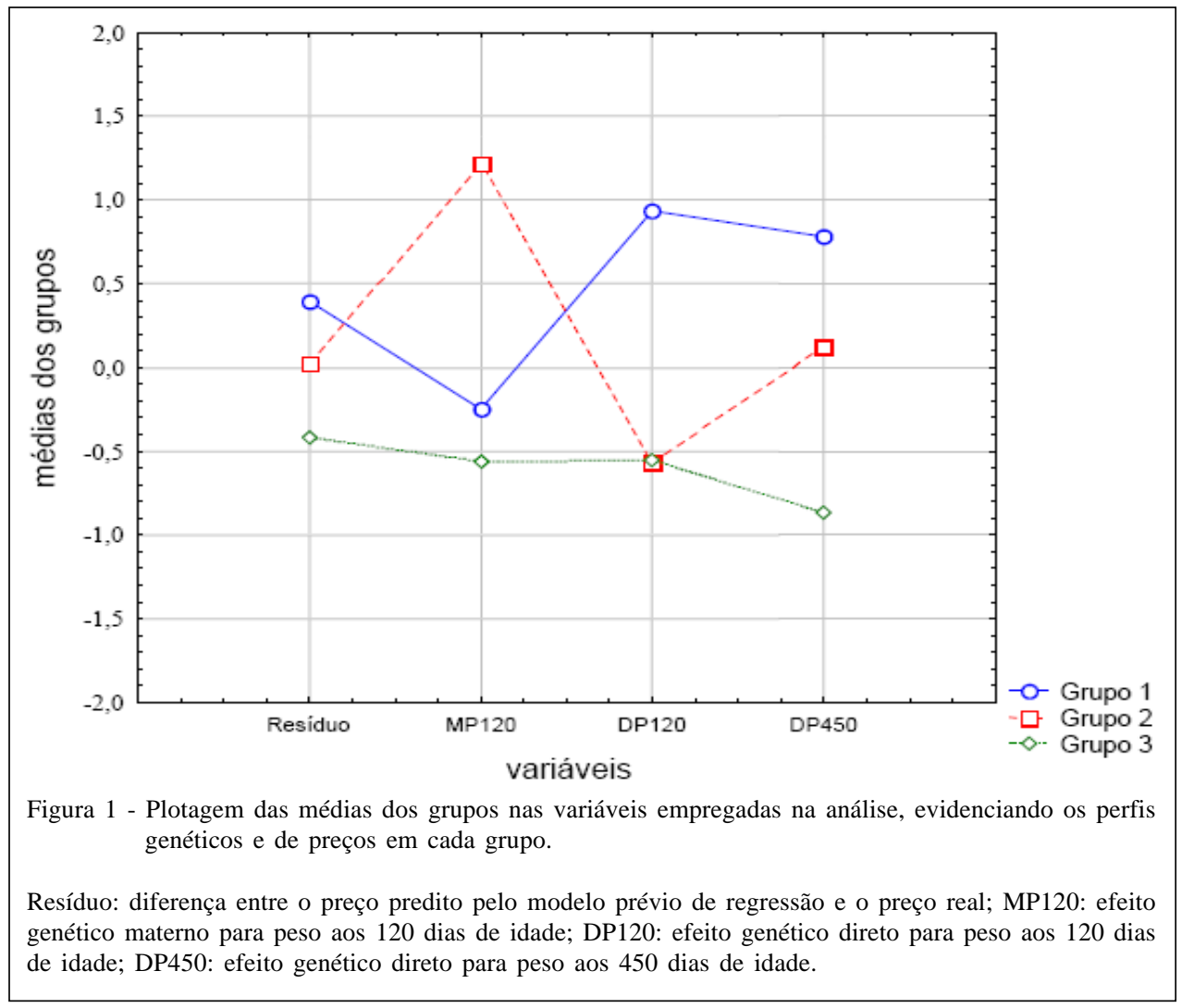

objetivo de seleção de criações de bovinos de corte na Espanha. Os autores encontraram grande importância relativa do peso adulto de vacas, do desenvolvimento pré-desmame, incluindo efeito materno, e da fertilidade. Os resultados do presente estudo indicam que os compradores de gado elite no Brasil têm valorizado principalmente as características ligadas ao desenvolvimento ponderal, negligenciando as necessidades de manutenção de peso adulto não muito elevado e de melhoria das taxas de fertilidade dos rebanhos, que podem ser quesitos importantes em condições de restrição alimentar, condição essa que é comum nos rebanhos comerciais brasileiros.

Análise das pontuações dos julgamentos

A análise dos pontos médios dos reprodutores nos julgamentos revelou que não houve relação significativa entre as pontuações acumuladas pelos reprodutores em julgamentos e os preços médios de suas progênies em leilões. Isso indica que os critérios que levaram os animais à obtenção de boas pontuações não coincidiram significativamente com os critérios que levaram ao aumento dos preços de venda dos animais em leilões.
ROCHA et al. (2006) estudaram esse mesmo conjunto de dados e descobriram que há uma correlação desejável, de intensidade moderada, entre as pontuações dos reprodutores em julgamentos e suas DEPs ligadas a desenvolvimento ponderal. Assim, apesar de não haver correlação significativa entre as pontuações e os preços, a escolha de animais pelas pontuações não apresentaria efeito negativo sobre os preços. No entanto, essa não seria a melhor estratégia de seleção ao se pensar na valorização dos animais. A seleção por meio de DEPs de desenvolvimento ponderal seria certamente mais eficiente nesse sentido. HORIMOTO (2005) também encontrou correlações favoráveis de intensidade moderada entre escores visuais e crescimento.

\section{CONCLUSÕES}

Animais com superioridade genética para desempenho ponderal, indicada pelas suas DEPs nos pesos aos 120 e 450 dias de idades, foram valorizados quando comercializados em leilões. A superioridade genética para efeito materno sobre os pesos aos 120 dias de idade também exerceu influência positiva sobre os preços de comercialização dos animais. Nenhuma 
outra característica de importância econômica na produção comercial apresentou influência significativa sobre os preços. Além disso, não houve relação significativa entre as pontuações acumuladas pelos reprodutores em julgamentos e os preços médios de sua crias em leilões.

\section{AGRADECIMENTOS}

Os autores agradecem à Associação Nacional dos Criadores e Pesquisadores (ANCP), pelo fornecimento de parte dos resultados dos preços de vendas dos animais em leilões e pela divulgação on-line das DEPs dos reprodutores. À Associação Brasileira dos Criadores de Zebu (ABCZ), pela divulgação online dos resultados de julgamentos. Esse projeto recebeu apoio financeiro da Fundação de Amparo à Pesquisa do Estado de minas Gerais (FAPEMIG) e contou com bolsas de Iniciação Científica do Conselho Nacional de Desenvolvimento Científico e Tecnológico (PIBIC-CNPq) e do Programa de Apoio à Pesquisa da Universidade de Uberaba (UNIUBE).

\section{REFERÊNCIAS}

AMER, P. Definition of selection objectives for sustainable systems of animal production. In: REUNIÃO DA SOCIEDADE BRASILEIRA DE ZOOTECNIA, 40., 2003, Santa Maria, RS. Anais... Brasilia: SBZ, 2003. 1 CD-ROM.

BICHARD, M. Cost benefits of investing in animal breeding. In: SIMPÓSIO NACIONAL DE MELHORAMENTO ANIMAL, 1996, Ribeirão Preto, SP. Anais... Viçosa: SBMA, 1996. 303p. p. $10-20$.

FERNÁNDEZ-PEREA, M.T.; ALENDA JIMÉNEZ, R. Economic weights for a selection index in Avileña purebreed beef cattle. Livestock Production Science, v.89, p.223233, 2004.

GUTIÉRREZ, J.P. et al. Genetic relationships between calving date, calving interval, age at first calving and type traits in beef cattle. Livestock Production Science, v.78, p.215-222, 2002.
HAIR, J.F. et al. Análise multivariada de dados. 5.ed. Porto Alegre: Bookman, 2005. 593p.

HORIMOTO, A.R.V.R. Estimativas de parâmetros genéticos para escores de estrutura corporal (frame) em bovinos de corte da raça nelore. 2005. 88f. Dissertação (Mestrado em Zootecnia) - Curso de Pós-graduação em Zootecnia, Universidade de São Paulo.

KOURY FILHO, W. et al. Parâmetros genéticos para escore de umbigo e características de produção em bovinos da raca nelore. Arquivo Brasileiro de Medicina Veterinária e Zootecnia, v.55, n.5, p.594-598, 2003.

LÔBO, R.B. Avaliação genética de animais jovens, touros e matrizes. Ribeirão Preto: GEMAC-Depto de GenéticaFMRP-USP, 1999. 90p.

PEREIRA, F.J.A. Dados para gerar informações que atendam às demandas do mercado. In: SIMPÓSIO NACIONAL DE MELHORAMENTO ANIMAL, 2., 1998, Uberaba, MG. Anais... Viçosa: SBMA, 1998. 465p. p.271-273.

ROCHA, C.D. et al. Production and reproduction genetic trends of the nellore beef cattle selected through visual assessment in Brazil. In: WORLD CONGRESS ON GENETICS APPLIED TO LIVESTOCK PRODUCTION, 8., 2006, Belo Horizonte, MG. Proceedings... Belo Horizonte: Instituto Prociência, 2006. 1 CD-ROM; 43\%4 pol. Disponível também: <www.wcgalp8.org.br>

STATSOFT, INC. Statistica: livro base (data analylis software system, version 7.1.). São Caetano do Sul: Copyright Statsoft, 2003. 142p.

VAL, J.E. et al. Alternativas para seleção de touros na raça Nelore considerando características múltiplas de importância econômica. Arquivo Brasileiro de Medicina Veterinária e Zootecnia, v.60, n.3, p.705-712, 2008.

WOLFOVÁ, N.; NITTER, G. Relative economic weights of maternal versus direct traits in breeding schemes. Livestock Production Science, v.88, p.117-127, 2004. 